\title{
Atteinte cérébrale au cours d'une maladie de Wegener
}

\section{Central nervous system involvement in Wegener's granulomatosis}

\author{
F. Jouret ${ }^{\mathrm{a}}$, S. Noirhomme ${ }^{\mathrm{a}}$, C. Grandin ${ }^{\mathrm{b}}$, M. Lonneux ${ }^{\mathrm{c}}$, C. Godfraind ${ }^{\mathrm{d}}$, \\ M. Lambert ${ }^{\mathrm{a}}$, C. Lefebvre ${ }^{\mathrm{a}, *}$ \\ ${ }^{a}$ Service de médecine interne générale, cliniques universitaires de Saint-Luc, université catholique Louvain, avenue Hippocrate, 1200 Bruxelles, Belgique \\ ${ }^{\mathrm{b}}$ Service de radiologie, cliniques universitaires Saint-Luc, université catholique de Louvain, Bruxelles, Belgique \\ ${ }^{c}$ Service de médecine nucléaire, cliniques universitaires Saint-Luc, université catholique de Louvain, Bruxelles, Belgique \\ ${ }^{\mathrm{d}}$ Service d'anatomo-pathologie, cliniques universitaires Saint-Luc, université catholique de Louvain, Bruxelles, Belgique
}

Disponible sur Internet le 2 juillet 2008

\section{Résumé}

Introduction. - La granulomatose de Wegener $(\mathrm{GW})$ est une vascularite nécrosante systémique associée à la présence d'auto-anticorps de type c-ANCA. L'atteinte du système nerveux central dans la GW est rare et correspond à une vascularite intraparenchymateuse, à la formation in situ de granulomes cérébroméningés ou à une extension par contiguïté au départ de la sphère ORL.

Observation. - Nous rapportons ici le cas d'une GW nasosinusienne sévère compliquée d'une extension intracrânienne pseudotumorale, confirmée par l'examen histologique.

Conclusion. - L'apport de la tomographie par émission de positons, dans notre observation, est un nouvel exemple de l'intérêt de cette imagerie fonctionnelle dans le bilan d'activité et d'extension, voire le suivi de la GW.

(C) 2008 Publié par Elsevier Masson SAS.

\section{Summary}

Introduction. - Wegener's granulomatosis (WG) is a systemic necrotizing vasculitis associated with c-ANCA antibodies. The involvement of the central nervous system in WG is uncommon and usually caused by in situ vasculitis, intracranial granuloma formation or contiguous invasion from extracranial sites.

Case report. - Here, we report on a tumour-like expansion of a severe nasosinusal WG into the brain, which was confirmed by brain biopsy examination.

Conclusion. - The positivity of positron emission tomography in our observation supports the potential role of such functional imaging in the staging, as well as in the follow-up of WG.

(C) 2008 Publié par Elsevier Masson SAS.

Mots clés : Vascularite systémique ; Syncope ; Tomographie par émission de positons

Keywords: Vasculitis; Syncope; Positron emission tomography

\section{Introduction}

La granulomatose de Wegener (GW) est une vascularite nécrosante systémique associée à la présence d'auto-anticorps dirigés contre le cytoplasme des polynucléaires neutrophiles (c-ANCA de type PR3). La maladie se manifeste habituellement par une atteinte de la sphère ORL, respiratoire et rénale

\footnotetext{
* Auteur correspondant.

Adressee-mail : chantal.lefebvre@uclouvain.be (C. Lefebvre).
}

de sévérité variable. Le diagnostic repose sur l'examen histologique d'une biopsie orientée montrant une angéite nécrosante et granulomateuse à cellules géantes [1]. Les manifestations neurologiques de la GW concernent 22 à $54 \%$ des patients et sont principalement de nature périphérique [2]. En revanche, l'atteinte du système nerveux central (SNC) est rare et estimée entre 4,8 et $15 \%$ [3]. Elle est soit associée à vascularite intraparenchymateuse soit à une localisation granulomateuse cérébroméningée, mais l'extension par contiguïté d'une atteinte nasosinusienne est le mécanisme le plus souvent rencontré, comme dans l'observation ci-après. 


\section{Observation}

Un homme de 52 ans était hospitalisé dans le service de médecine interne générale pour exploration d'une chute sans prodrome avec perte de connaissance. Le patient décrivait des céphalées frontales et maxillaires accompagnées de douleurs péri-orbitaires bilatérales depuis plusieurs mois. Récemment, une symptomatologie digestive haute associant nausées et vomissements était apparue. Le jour de son admission, le patient tombait et perdait connaissance pendant environ une minute. Son épouse décrivait une phase post-critique où le patient était resté obnubilé, dysarthrique et pâle pendant une vingtaine de minutes. L'antécédent principal du patient était une GW nasosinusienne diagnostiquée à l'âge de 40 ans devant un tableau de nodules pulmonaires nécrotiques récidivants et de perforation de la cloison nasale, associé à des sécrétions mucopurulentes et sanguinolentes chroniques. La sérologie c-ANCA de type PR3 était alors positive et la biopsie de la cloison nasale suggestive d'une GW. Un traitement immunosuppresseur, associant cyclophosphamide et corticoïdes selon un schéma dégressif était instauré, laissant comme séquelle la disparition complète de la cloison nasale et la destruction des sinus maxillaires, frontaux et sphénoïdaux. Cet état clinique était stable depuis plus de dix ans, avec un traitement comportant de la méthylprednisolone à la dose quotidienne de $8 \mathrm{mg}$ en association avec du sulfaméthoxazole $(800 \mathrm{mg})$ et du triméthoprime $(160 \mathrm{mg})$.

À l'admission, l'examen clinique était normal : pouls à 58 par minute, pression artérielle à $130 / 80 \mathrm{mmHg}$ et température à $36,9^{\circ} \mathrm{C}$. Aucun déficit neurologique n'était retrouvé, mais le patient se plaignait de photophobie et d'hyperacousie. Les examens biologiques montraient une neutrophilie (16000 par $\mathrm{mm}^{3}$ ) secondaire à la corticothérapie, en l'absence de tout syndrome inflammatoire (CRP inférieur à $\left.10 \mathrm{mgL}^{-1}\right)$. La fonction rénale était normale. La recherche de c-ANCA était négative. La radiographie conventionnelle du thorax montrait une image stellaire apicale droite de type séquellaire. L'électrocardiogramme objectivait une bradycardie sinusale (53 par minute) sans anomalies du complexe QRS. La tomodensitométrie cérébrale retrouvait un remaniement majeur des fosses nasales et des sinus transformés en une cavité unique avec un important épaississement osseux (Fig. 1A). En outre, une masse intracrânienne paramédiane droite relativement hyperdense (39 unités hounsfield [UH]) de $20 \times 15 \times 25 \mathrm{~mm}$ était mise en évidence au contact d'un déficit osseux de la lame criblée. Cette masse était entourée d'un vaste œdème vasogénique dans le lobe frontal droit. L'imagerie par résonance magnétique confirmait la présence de cette masse intraparenchymateuse en l'absence de toute réaction méningée de voisinage, compatible avec une extension intracrânienne de la GW sinusienne (Fig. 1B). Une imagerie par tomographie par émission de positons (TEP) après injection de 18-fluoro-déoxy-glucose (18-FDG) révélait un foyer nodulaire hypermétabolique (standardized uptake value [SUV] à 14,9) au niveau de la masse cérébrale identifiée en radiologie standard (Fig. 2A). En outre, on notait plusieurs nodules hyperfixants au niveau des apex pulmonaires, compatibles avec des lésions actives de la GW (Fig. 2B-D).

Afin d'exclure une étiologie infectieuse ou néoplasique, une biopsie stéréotaxique de la masse frontale était réalisée sous neuronavigation (Fig. 3). L'examen histologique de ces fragments de parenchyme cérébral montrait un infiltrat inflammatoire riche en lymphocytes B au sein des parois vasculaires, associé à une gliose lymphocytaire. La réalisation de coupes semi-sériées ne mettait pas en évidence de nécrose fibrinoïde ou granulomateuse au sein de la paroi des vaisseaux. La recherche de la protéine acide fibrillaire gliale (GFAP) et du proto-oncogène p53 était négative. Les colorations de PAS et de Grocott ne démontraient pas d'agent mycotique.

Étant donné l'aspect histologique évocateur d'une vascularite et l'absence d'argument en faveur d'une pathologie infectieuse ou tumorale, le traitement immunosuppresseur était renforcé par une corticothérapie (méthylprednisolone $2 \times 1 \mathrm{~g}$ intraveineux, relais $32 \mathrm{mg} / \mathrm{j}$ per os) en association à des bolus intraveineux mensuels de cyclophosphamide $\left(1 \mathrm{~g} / \mathrm{m}^{2}\right)$ et à la poursuite du sulfaméthoxazole $(800 \mathrm{mg})$ et du triméthoprime $(160 \mathrm{mg})$ à visée préventive anti-staphylococcique et anti-Pneumocystis jiroveci. Une couverture anti-épileptique à base d'acide valprö̈que était
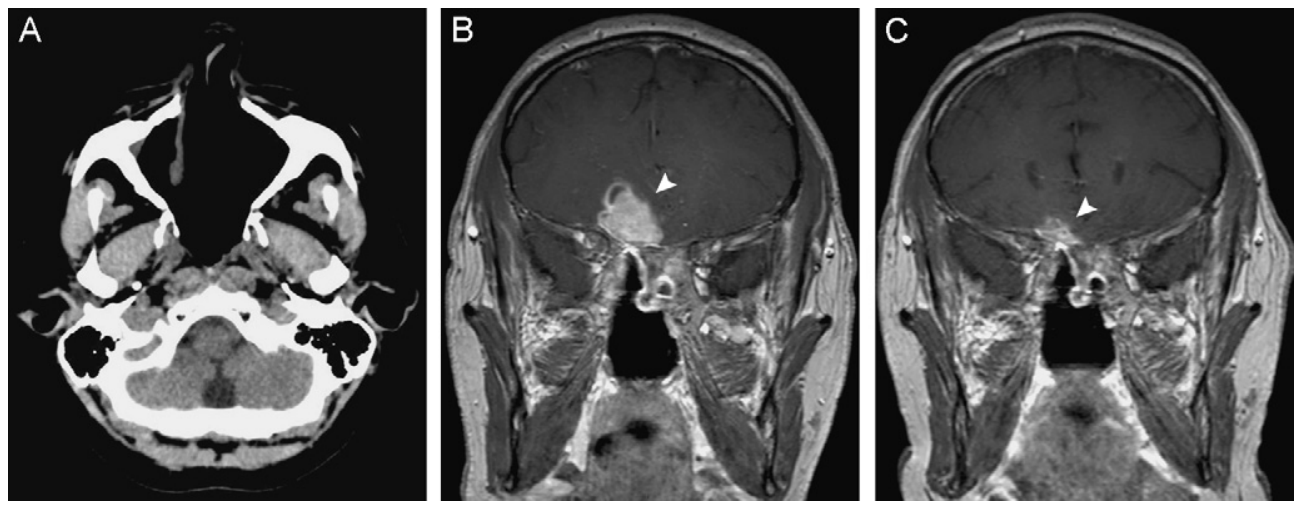

Fig. 1. Imagerie par tomodensitométrie et résonance magnétique cérébrales.

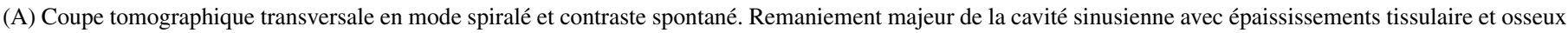

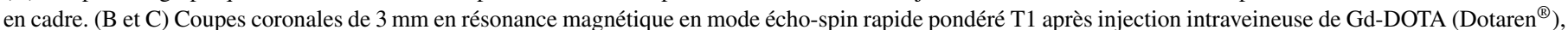

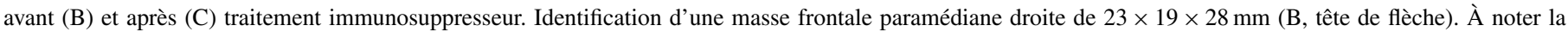

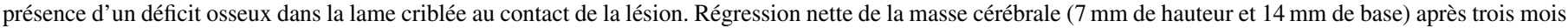
d'immunosuppression ( $C$, tête de flèche). 

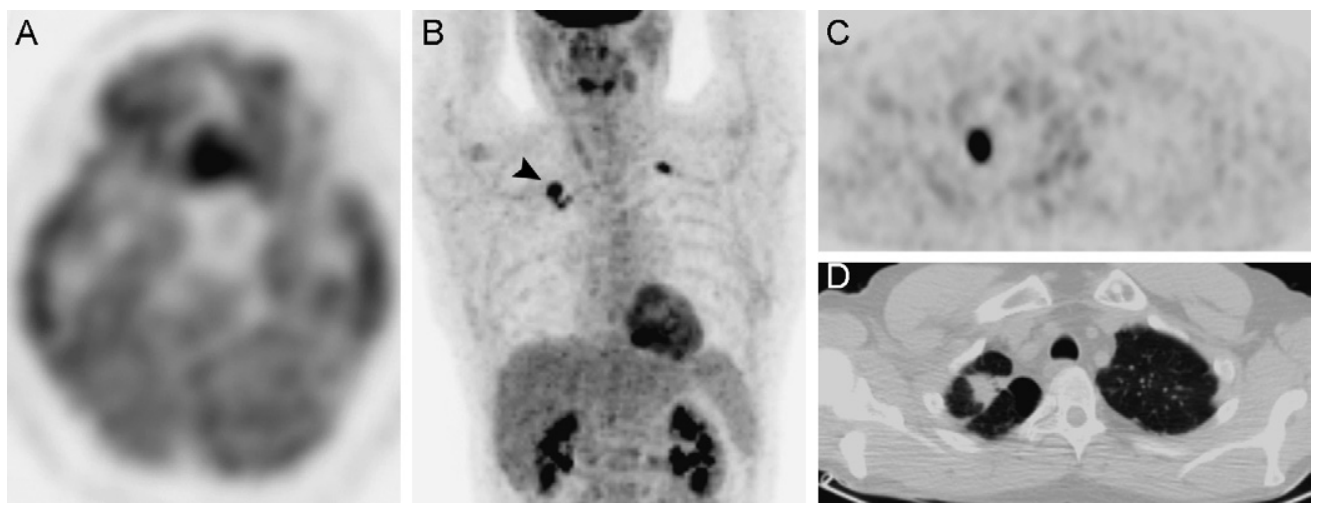

Fig. 2. Imagerie par tomographie par émission de positons après injection de 18-fluoro-déoxy-glucose.

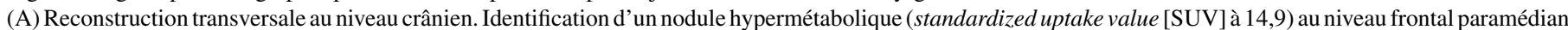

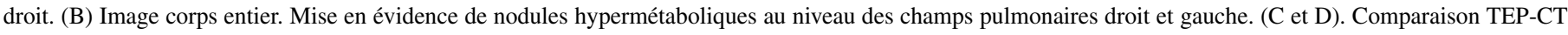
après reconstruction transversale des apex pulmonaires à hauteur du nodule pulmonaire droit (B : tête de flèche).
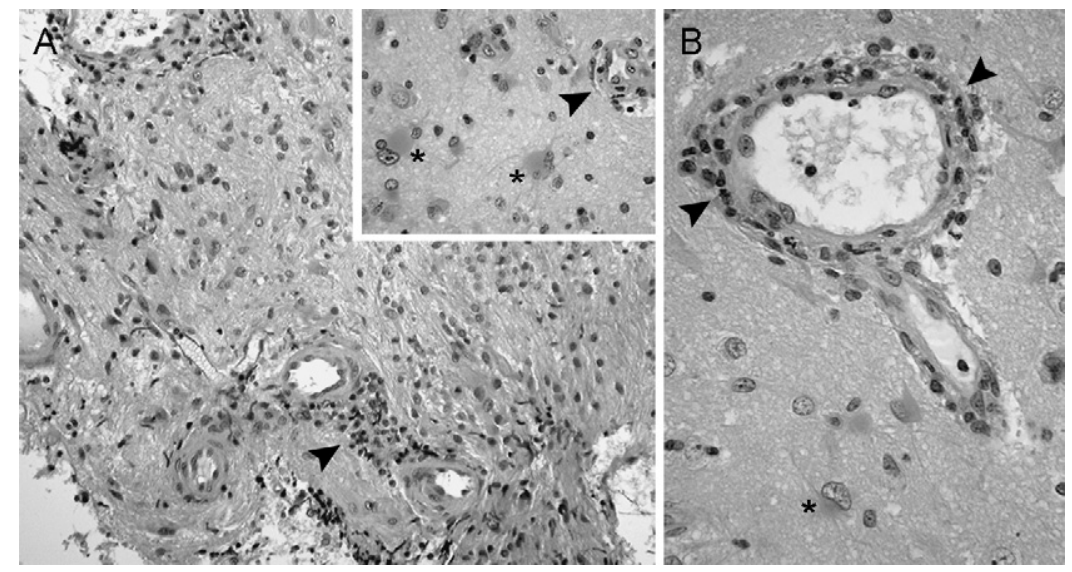

Fig. 3. Examen anatomopathologique de la biopsie cérébrale.

(A) Faible grossissement (A : objectif $10 \times$ ), observation d'une gliose réactionnelle lymphocytaire, de vaisseaux aux parois épaissies par un infiltrat cellulaire riche en lymphocytes B (A : tête de flèche) et d'astrocytes réactionnels (inset, *). A plus fort grossissement (B : objectif 40x), illustration de la vascularite à infiltrat lymphocytaire et de l'astrocytose (*). Coloration à l'hématoxiline-éosine.

également débutée. Le suivi à trois mois montrait une résolution complète des douleurs faciales et des symptômes d'hypertension intracrânienne, et révélait une régression spectaculaire de la masse intracrânienne à l'imagerie par résonance magnétique (Fig. 1C).

\section{Discussion}

Notre observation illustre l'extension intracérébrale d'une GW nasosinusienne ancienne. La présentation clinique était brutale, bien que l'anamnèse détaillée révélait le développement à bas bruit d'une hypertension intracrânienne. La biologie sanguine était pauvre. En revanche, l'imagerie cérébrale, en particulier la résonance magnétique, démontrait l'extension intraparenchymateuse de la GW au travers de la lame criblée. En outre, la TEP nous renseignait sur l'activité globale de la maladie, identifiant une atteinte pulmonaire silencieuse, en plus de la masse frontale. Enfin, l'analyse histologique confirmait la nature vasculitique de la lésion cérébrale, justifiant le renforcement du traitement immunosuppresseur. L'évolution clinique rapidement favorable avec régression complète des signes neurologiques confortait notre diagnostic et écartait rétrospectivement une atteinte infectieuse ou tumorale [3].

L'atteinte neurologique dans la GW est rapportée chez 22 à $54 \%$ des patients [2]. Sa prévalence est plus importante chez le sujet âgé, de sexe masculin et dans les formes diffuses de la maladie caractérisées par un titre élevé de c-ANCA. Elle affecte principalement les nerfs périphériques. Cette neuropathie se présente sur le plan clinique et électromyographique par une monoou une multi-névrite, d'abord sensitive, puis mixte, et asymétrique [4,5]. D' un point de vue histologique, l'atteinte neurogène périphérique est la conséquence d'une vascularite des vasa nervorum. Malgré un traitement immunosuppresseur bien conduit, la récupération est souvent modeste et inconstante. À l'inverse, l'atteinte neurologique centrale dans la GW ne touche que 4 à $15 \%$ des patients selon les séries, et est rarement révélatrice de la maladie [3,4]. Trois mécanismes physiopathologiques différents peuvent rendre compte de l'atteinte du SNC dans la GW [6] :

- la vascularite;

- l'extension par contiguïté du tissu granulomateux à partir des fosses nasales ou des sinus ; 
- la formation in situ d'un granulome au sein du parenchyme cérébral ou des méninges.

La vascularite intracérébrale se manifeste habituellement sous la forme d'un accident vasculaire cérébral de type ischémique ou hémorragique. L'effet de masse de l'inflammation granulomateuse entraîne une symptomatologie d'hypertension intracrânienne typique, parfois compliquée d'une perte de connaissance, comme décrit ici.

L'imagerie cérébrale par résonance magnétique est l'examen de choix dans la démarche diagnostique d'une atteinte neurologique centrale associée à la GW [3]. Une étude systématique portant sur 19 patients atteints de GW compliquée d'une atteinte neurologique centrale montrait un épaississement méningé, diffus ou focalisé, dans plus de la moitié des cas [7]. À noter la présence d'hypersignaux en pondération T2 d'allure non spécifique et un élargissement de la tige pituitaire chez dix et deux patients, respectivement. Les autres méthodes classiques d'exploration du SNC, telles que l'électroencéphalogramme, l'analyse du liquide céphalorachidien et l'angiographie sélective, n'apportent pas d'éléments diagnostiques décisifs dans l'atteinte cérébrale de la GW.

La TEP, récemment couplée à la résolution tomodensitométrique (TEP-CT), permet de réaliser une imagerie fonctionnelle du corps entier grâce à l'utilisation de différents traceurs, en particulier le 18-FDG. Véritable cartographie biochimique in vivo, cette technique a connu un rapide essor dans le domaine de l'oncologie en améliorant le bilan d'extension tumorale et, partant, la prise en charge du patient. De nouvelles applications du TEP-CT se développent actuellement, notamment dans le cadre de la mise au point diagnostique d' une vascularite des gros troncs ou d'une fièvre nue au long cours [8,9]. En effet, le traceur 18FDG s'accumule non seulement au niveau des cellules malignes et du stroma tumoral, mais aussi au niveau des sites d'infection [10] ou d'inflammation au sens large tels que rencontrés dans les maladies auto-immunes [11]. Plus particulièrement, les données préliminaires de la littérature actuelle montrent une sensibilité de 77 à $92 \%$ et une spécificité de 89 à $100 \%$ du TEP-CT dans le diagnostic de vascularite à cellules géantes chez un patient atteint d'un syndrome inflammatoire biologique [12]. La place du TEP-CT dans la démarche diagnostique des autres vascularites telles que la GW, le syndrome de Churg-Strauss et la périartérite noueuse est actuellement en cours d'évaluation. La rentabilité de l'examen semble liée à l'atteinte des gros troncs vasculaires et/ou des tissus parenchymateux adjacents [9]. Dans le cas particulier d'une atteinte inflammatoire ou tumorale du SNC, l'activité corticale, normalement intense à l'état basal, peut cependant masquer la lésion [13]. Le tissu neuronal est en effet très avide en FDG, le glucose constituant le substrat énergétique principal du cerveau. Dans notre observation, l'activité métabolique de la lésion vasculitique était toutefois supérieure à l'activité corticale basale et se manifestait par un signal positif net. En outre, l'identification de nodules hypermétaboliques au sein des deux parenchymes pulmonaires et du cerveau chez notre patient démontre l'intérêt du TEP-CT dans l'évaluation de l'activité, le bilan d'extension, voire le suivi d'une GW [14].
La preuve histologique est rarement obtenue lors d'une atteinte cérébrale de GW et l'initiation du traitement se fonde alors sur un faisceau d'arguments cliniques et d'imagerie. La présentation pseudotumorale décrite ici nécessitait cependant l'analyse histologique d'une biopsie de la masse intraparenchymateuse afin d'exclure un processus malin, même si, parmi les 79 vasculites systémiques associées à des lésions pseudotumorales rapportées par Kariv et al. [15], la GW était la cause la plus fréquente. En outre, le SNC représente, après le sein, la deuxième localisation préférentielle de pseudotumeurs dans la GW. L'identification précoce de la nature vasculitique de ces lésions par des arguments cliniques, sérologiques, radiologiques et histologiques permet non seulement d'éviter un geste chirurgical inutile et risqué, mais aussi l'instauration rapide d'un traitement immunosuppresseur adéquat. L'attitude thérapeutique face à une localisation intracérébrale de la GW consiste en une corticothérapie à hautes doses associée à des médicaments immunosuppresseurs de type cyclophosphamide. Le relais thérapeutique utilise le méthotrexate ou l'azathioprine. La sensibilité au traitement, marquée par une résolution rapide et complète des signes neurologiques, conforte le diagnostic d'atteinte cérébrale de $\mathrm{GW}$, et permet, rétrospectivement, d'exclure toute autre étiologie mimant ce tableau clinique, telle qu'une atteinte infectieuse, athéromateuse ou tumorale. Bien que certains auteurs rapportent une évolution péjorative en l'absence d'c-ANCA, le pronostic est habituellement favorable [3].

\section{Remerciements}

Les auteurs remercient l'équipe de Neurochirurgie du Pr. Ch. Raftopoulos et, en particulier, le Dr M. Delavallée, pour leur collaboration indispensable à la mise au point diagnostique. Que P. Malengreau soit ici remerciée pour son travail de dactylographie et de mise en page.

\section{Références}

[1] Rao JK, Weinberger M, Oddone EZ, Allen NB, Landsman P, Feussner JR. The role of antineutrophil cytoplasmic antibody (c-ANCA) testing in the diagnosis of Wegener granulomatosis. A literature review and metaanalysis. Ann Intern Med 1995;123:925-32.

[2] Seror R, Mahr A, Ramanoelina J, Pagnoux C, Cohen P, Guillevin L. Central nervous system involvement in Wegener granulomatosis. Medicine (Baltimore) 2006;85:54-65.

[3] Konaté A, Le Falher G, Crozat-Grosleron S, Rivière S, Le Quellec A. Incidence and presentation of the central neurological manifestations of Wegener's granulomatosis: a monocentric study of 14 cases. Rev Med Interne 2004;25:183-8.

[4] Nishino H, Rubino FA, Parisi JE. The spectrum of neurologic involvement in Wegener's granulomatosis. Neurology 1993;43:1334-7.

[5] de Groot K, Schmidt DK, Arlt AC, Gross WL, Reinhold-Keller E. Standardized neurologic evaluations of 128 patients with Wegener granulomatosis. Arch Neurol 2001;58:1215-21.

[6] Silvera S, Vignaux O, Legmann P. Sinonasal and cerebral imaging findings in Wegener's granulomatosis. Presse Med 2007;36:913-21.

[7] Murphy JM, Gomez-Anson B, Gillard JH, Antoun NM, Cross J, Elliott JD, et al. Wegener granulomatosis: MR imaging findings in brain and meninges. Radiology 1999;213:794-9.

[8] Raynaud FR, Huglo D, Steinling M. Positron emission tomography: current use in internal medicine and future perspectives. Rev Med Interne 2006;27:932-45. 
[9] Meller J, Sahlmann CO, Scheel AK. 18F-FDG PET and PET/CT in fever of unknown origin. J Nucl Med 2007;48:35-45.

[10] Dumarey N, Egrise D, Blocklet D, Stallenberg B, Remmelink M, del Marmol V, et al. Imaging infection with 18F-FDG-labeled leukocyte PET/CT: initial experience in 21 patients. J Nucl Med 2006;47:625-32.

[11] Jaruskova M, Belohlavek O. Role of FDG-PET and PET/CT in the diagnosis of prolonged febrile states. Eur J Nucl Med Mol Imaging 2006;33:913-8.

[12] Blockmans D, de Ceuninck L, Vanderschueren S, Knockaert D, Mortelmans L, Bobbaers H. Repetitive 18F-fluorodeoxyglucose positron emission tomography in giant cell arteritis: a prospective study of 35 patients. Arthritis Rheum 2006;55:131-7.

[13] Sossi V. Cutting-edge brain imaging with positron emission tomography. Neuroimaging Clin N Am 2007;17:427-40.

[14] Vandergheynst F, Goldman S, Cogan E. Wegener's granulomatosis overlapping with Takayasu's arteritis revealed by FDG-PET scan. Eur J Intern Med 2007;18:148-9.

[15] Kariv R, Sidi Y, Gur H. Systemic vasculitis presenting as a tumorlike lesion. Four case reports and an analysis of 79 reported cases. Medicine (Baltimore) 2000;79:349-59. 